Cite this: Phys. Chem. Chem. Phys., 2011, 13, 9578-9589

\title{
Implicit numerical schemes for the stochastic Liouville equation in Langevin form
}

\author{
Pär Håkansson ${ }^{* a}$ and Prasanth B. Nair $\dagger^{b}$ \\ Received 15th February 2011, Accepted 17th March 2011 \\ DOI: $10.1039 / \mathrm{c1cp20400a}$
}

We present and numerically test implicit as well as explicit numerical schemes for solving the Stochastic Liouville Equation in Langevin form. It is found that implicit schemes provide significant gain in robustness, for example, when nonsecular Hamiltonian terms cannot be ignored in electron and nuclear spin resonance. Implicit schemes open up several spectroscopic relaxation problems for direct interpretation using the Stochastic Liouville Equation. To illustrate the proposed numerical schemes, studies are presented for an electron paramagnetic resonance problem involving a coordinated copper complex and a fluorescence problem.

\section{Introduction}

The Stochastic Liouville Equation in Langevin form (SLEL) provides a rigorous description of interacting quantum degrees of freedom coupled to a surrounding thermal bath. The SLEL is an attractive tool for interpreting spectroscopic experiments since significant physical insights can be gained at the molecular level. ${ }^{1}$ A seminal work on SLE and molecular fluctuations is by $\mathrm{Kubo}^{2}$ and is more recently applied in the study of a wide set of relaxation phenomena. ${ }^{3}$ However, the computational cost associated with solving the SLEL in the time domain or the Stochastic Liouville Equation (SLE) in the frequency domain is generally high. As a result, most of the work in this topic has focused on efficient methods for small quantum systems. In Electron Spin Resonance (EPR), a lineshape for a nitroxide spinprobe is efficiently computed by solving the SLE in the frequency domain by Schnider and Freed. ${ }^{4}$ For the same problem, computing a lineshape observable using the SLEL is a higher but still a relatively low/acceptable cost calculation. ${ }^{5}$ A possible reason for this low computational cost is that a high-field approximation is applicable, i.e., the so called nonsecular terms can be removed from the spin Hamiltonian. We discuss the format of nonsecular approximation used in the present work in Appendix B. We note that for a transition metal complex in EPR, the high-field approximation is not applicable. ${ }^{6}$ This approximation in time domain calculations effectively removes a fast oscillating Larmor frequency

${ }^{a}$ Computational Engineering and Design Group,

School of Engineering Sciences, University of Southampton,

Highfield, United Kingdom. E-mail: P.Hakansson@soton.ac.uk

${ }^{b}$ Computational Engineering and Design Group,

School of Engineering Sciences, University of Southampton,

Highfield, United Kingdom.E-mail: P.B.Nair@soton.ac.uk

$\dagger$ Present address: University of Toronto, Institute for Aerospace Studies, 4925 Dufferin Street, Ontario, Canada M3H 5T6, Email: pbn@utias.utoronto.ca from the problem and also reduces the dimensionality of the Liouville matrix. Numerical experience gained from recent work on Nuclear Magnetic Resonance (NMR) ${ }^{7}$ and a fluorescence dipolar interaction problem ${ }^{8}$ suggests that the computational cost required for solving the SLEL increases substantially when a fast relaxation rate is present in the problem. More specifically, for the NMR problem, it was the inclusion of nonsecular terms and a high-field that increased the cost substantially. The fluorescence problem contains a fast relaxation rate and hence when the observable relaxes slowly the computational burden becomes large due to the requirement of employing a small timestep.

The above observations motivate further work on computational methods for solving the SLEL and an exploration of implicit numerical schemes that have so far never have been tested for the SLEL type problems we consider here. With implicit, we mean that the density operator is approximated at timestep $n+1$ as $\rho_{n+1}=f\left(\rho_{n+1}\right)$-in other words, a system of equations need to be solved at each timestep. The objective of the present work is to examine carefully if implicit numerical schemes can resolve some of the computational bottlenecks that arise when a fast relaxation rate is present in the problem under consideration.

We note that in the majority of existing work on this topic, the starting assumption is a high-field approximation form of the original Liouville problem or the problem is recast as a stochastic Schrödinger equation. ${ }^{9-14}$ In this context, we note that a recently developed low computational cost method ${ }^{15}$ addresses a transverse relaxation process in EPR, not including non-secular terms and as such it involves the same level of approximation as the approaches mentioned earlier. The only problems we are aware of within magnetic resonance and using the SLEL in the presence of nonsecular terms is the NMR problem ${ }^{7}$ discussed earlier, with a $9 \times 9$ Liouville matrix and a $S>1 / 2$ EPR problem ${ }^{16}$ (up to $64 \times 64$ Liouville matrix). 
In both studies, an explicit propagator in either exponential or Taylor expanded form is employed. Similarly, the $S>1 / 2$ EPR problem as treated in ref. 16 has been studied in ref. 17, but cast in a stochastic Shrödinger form and propagation in time is carried out by Taylor expanding a propagator at each timestep. From a mathematical perspective, a similar problem is time-dependent quantum mechanical calculations for molecular dynamics, ${ }^{18}$ and variety of numerical approaches have been studied in that context. For instance, Taylor expansions are suggested to be unstable while formulating numerical schemes for such problems, ${ }^{18}$ whereas for SLEL problems a Taylor construction has been found to work well in several problems. ${ }^{16,19}$ This motivates us to include a Taylor form in the presented numerical study and see if this can lead to an efficient computational method.

In contrast to quantum mechanical molecular dynamics type of problems, ${ }^{18}$ the SLEL depends on a stochastic process. That motivates us to discuss the derivation of numerical schemes within the framework of Itô-calculus. ${ }^{20}$ We discuss the SLEL problem in a common format as the underlying stochastic process at the molecular level, since we believe that this representation is valuable in capturing the global error of the simulation.

In this paper, we first discuss the format of the SLEL in magnetic resonance and outline how previously used approaches is interpreted within the framework of Itô-calculus. Subsequently, in section 2 , we propose implicit numerical schemes and outline its properties. In section 3, we explore implicit and explicit numerical schemes for problems with Liouville matrix of sizes $36 \times 36,5184 \times 5184$, and $4 \times 4$, where the first two problems are EPR problems while the final example involves a fluorescence dipolar interaction problem. It is found that one of the implicit schemes tested conserves the norm of the density operator, but still has similar cost as a Taylor expansion type of scheme. Finally, in section 3.3, we calculate for the first time EPR observable of electron spin coupled to copper and two nitrogen nucleus to provide a new chemically relevant problem addressed with the SLEL.

\subsection{Problem definition}

In previous works on $\mathrm{EPR}^{5,16,21,22}$ and $\mathrm{NMR}^{7}$ problems, the following SLEL is numerically solved

$$
\frac{\mathrm{d}}{\mathrm{d} t} \rho\left(\Omega_{t}\right)=-i\left[\mathscr{L}_{S}+\mathscr{L}_{S L}\left(\Omega_{t}\right)\right] \rho\left(\Omega_{t}\right)
$$

where $\mathscr{L}_{\mathrm{X}} \equiv\left[H_{X}, \cdot\right], X=$ " $S$ ",' $L S$ ' are the superoperators. $H_{S}$ and $H_{S L}\left(\Omega_{t}\right)$ are the Hamiltonians representing the isolated coherent quantum subsystem (S) and the incoherent spin-lattice coupling dependent on stochastic process in time $\left(\Omega_{t}\right)$, respectively. $\rho\left(\Omega_{t}\right)$ is a reduced finite-dimensional density operator for quantum degrees of freedom and $i=\sqrt{-1}$. For numerical purposes, it is convenient to specify a basis and express eqn (1) in matrix form. Using an orthonormal basis $\left[Q_{1}, Q_{2}, \ldots, Q_{M}\right]$, we get

$$
\begin{aligned}
\rho_{k l} & =\rho_{i}=\left(Q_{i} \mid \rho\right), \\
L_{k l, m n} & =L_{i j}=\left(Q_{i}|\mathscr{L}| Q_{j}\right),
\end{aligned}
$$

where a scalar product $(A \mid B) \equiv \operatorname{Tr}\left(A^{\dagger} B\right)$ is used. ${ }^{23}$ It is useful in practice to transform the numerical problem to an interaction picture $(I)$ with the transformations

$$
\begin{aligned}
U_{S}\left(t, t_{0}\right) & =\exp \left(-i L_{S}\left(t-t_{0}\right)\right), \\
\rho_{I}(t) & =U_{S}^{\dagger}\left(t, t_{0}\right) \rho(t), \\
L_{I}\left(\Omega_{t}\right) & =U_{S}^{\dagger}\left(t, t_{0}\right) L_{S L}\left(\Omega_{t}\right) U_{S}\left(t, t_{0}\right) .
\end{aligned}
$$

where $t_{0}$ denotes the initial time.

\subsection{SLEL in Itô calculus format}

In this section, we formulate a numerical scheme for solving the SLEL in Itô format with molecular dynamics represented in the form of a diffusion process modelling the relevant fluctuations in the spin Hamiltonian. To arrive at numerically tractable forms, Itô-Taylor expansions will be formulated. The present derivation accounts for the diffusion process and the Liouville state time evolution on an equal footing. The objective behind this is to find an efficient and general numerical scheme for the complete solution. Treating the SLEL and diffusion on an equal footing is slightly more involved compared to considering them individually, but the former approach provides valuable theoretical and practical information.

The format for Brownian molecular dynamics in Itô calculus formalism is to a large extent already developed. ${ }^{20,24,25}$ The format developed here enables subsequent discussion of frequently used short time SLEL solution in exponential form as well as alternative numerical schemes. The somewhat elaborate discussion below is further motivated by the rigorous mathematical foundation of Itô calculus where in particular the existence of an error expansion enables extrapolation methods to be used - this is valuable when dealing with high dimensional problems ${ }^{26}$ and is a direction we will take in future work on the SLEL. Molecular dynamics in the form of a constrained Brownian motion expressed as an Itô diffusion $^{25}$ has been found applicable in several problems $s^{7,16,27}$ and we refer to these papers for specific examples.

Consider the following formal integration of the SLEL and diffusion process,

$$
\begin{aligned}
\rho_{I}\left(\Omega_{t}\right) & =\rho_{I}\left(\Omega_{0}\right)-i \int_{0}^{t} L_{I}\left(\Omega_{s}\right) \rho_{I}\left(\Omega_{s}\right) \mathrm{d} s, \\
X_{t} & =X_{0}+\int_{0}^{t} A\left(X_{s}\right) \mathrm{d} s+\int_{0}^{t} B\left(X_{s}\right) \mathrm{d} W_{s}, \\
\Omega_{s} & \equiv \Omega\left(X_{s}\right),
\end{aligned}
$$

where eqn (7) is in the interaction picture and eqn (9) represents a mapping between Cartesian coordinates and the rotation matrix elements (see Appendix B). It is a formal representation since we do not know the time evolution of $\rho_{I}\left(\Omega_{s}\right)$ and $X_{S}$ needed to evaluate the above integrals. $X_{t}$ is an Itô diffusion in Cartesian coordinates and a Brownian motion $\left(W_{t}\right)$ with drifts vector $A$ and diffusion matrix $B$ taking care of constraints in a rigorous way. ${ }^{25,28}$ The suitable choice of molecular dynamical process in eqn (8) is problem specific, however, often a low dimension $m$ of $W_{t}$ can be useful. For instance, Brownian motion on a sphere with $W_{t} \in \mathbb{R}^{3}$ 
(representing a spherical shaped molecule in solution) or in a constraining potential. ${ }^{27}$

We may proceed with the numerical solution and a short time form of eqn (7)-(8) with the explicit Euler scheme ${ }^{20}$ (henceforth denoted by E1) as shown below.

$$
\begin{aligned}
\tilde{\rho}_{I}\left(\Omega_{n+1}\right) & =\left[\mathscr{E}-i L_{I}\left(\Omega_{n}\right) \Delta t\right] \tilde{\rho}_{I}\left(\Omega_{n}\right), \\
\tilde{X}_{n+1}^{k} & =\tilde{X}_{n}^{k}+a^{k}\left(\tilde{X}_{n}\right) \Delta t+\sum_{j=1}^{m} b^{k, j}\left(\tilde{X}_{n}\right) \Delta W^{j},
\end{aligned}
$$

where (.) indicates "numerically computed" quantities, $\tilde{\rho}_{I}\left(\Omega_{n+1}\right)$ is in vector format (dimension $M$ ) and the $k$ th component of $\tilde{X}_{n+1}($ dimension $m$ ) is listed, and $\mathscr{E}$ is the unity matrix. The independent Brownian processes $W^{j}, j=1, \ldots, m$ has mean zero and variance $\overline{\left(\Delta W^{j}\right)^{2}}=2 D \Delta t$, where $D$ is a diffusion constant. A relevant observable is obtained by a projection of $\tilde{\rho}_{I}\left(\Omega_{n+1}\right)$; for example, an EPR lineshape observable is given by $\overline{\tilde{\rho}_{\text {obs }}\left(\Omega_{n}\right)}=\overline{\operatorname{Tr}\left\{S_{+}^{(1)} \tilde{\rho}_{I}\left(\Omega_{n}\right)\right\}}$, where $(\overline{\{.\}})$ denotes the ensemble average. With a discretisation of time into $N+1$ points with $\Delta t=T / N$ we can compute the observable over the the time window $[0, T]$ using the following steps:

1. Generate an initial orientation $\left(\Omega_{0}\right)$ from the distribution $P\left(\Omega_{0}\right)$,

2. Numerically solve eqn (11) and compute $\left(\Omega_{n}\right)$ at $n=0$, $1, \ldots, N$,

3. Numerically solve eqn (10) for $\tilde{\rho}_{\text {obs }}\left(\Omega_{n+1}\right)$ at $n=0$, $1, \ldots, N$,

4. Repeat steps 1-3L times and compute $\mathscr{A}\left(\tilde{\rho}_{\text {obs }}\left(\Omega_{n}\right), L\right)$ as follows:

$$
\mathscr{A}\left(\tilde{\rho}_{\mathrm{obs}}\left(\Omega_{n}\right), L\right)=\frac{1}{L} \sum_{i=1}^{L} \tilde{\rho}_{\text {obs }}\left(\Omega_{n}\right) .
$$

In summary, the solution is calculated $L$ times, where $L$ is chosen such that the statistical error in the observable is sufficiently small.

We now proceed to consider a relevant error metric for an arbitrary ordered $\beta$ weak Itô-Taylor scheme. It is worth noting here that the scheme $\mathbf{E} 1$ outlined earlier corresponds to $\beta=1$. We are interested in accurate expectation values and hence weak Itô-Taylor approximations are considered. The approximate solution with $\Delta t \in\left(0, \Delta t_{\max }\right), \tilde{\rho}_{\text {obs }}^{\Delta t}$, is said to converge weakly to $\rho_{\text {obs }}$ with order $\beta>0$ if for each observable there exists a constant $C_{\text {obs }}^{T}$ independent of $\Delta t$ such that

$$
|\operatorname{dev}(T, \Delta t)| \leq C_{\mathrm{obs}}^{T}(\Delta t)^{\beta},
$$

where

$$
\operatorname{dev}(T, \Delta t)=\overline{\rho_{\mathrm{obs}}(T)}-\overline{\tilde{\rho}_{\mathrm{obs}}^{\Delta t}(T)},
$$

for all $\Delta t \in\left(0, \Delta t_{\max }\right)$. Thus in terms of the error bound in eqn (13), for the explicit Euler scheme E1, we have $\beta=1$.

Using the weak Itô-Taylor approximation, an explicit scheme with $\beta=2$ is given by. ${ }^{20}$ :

$$
\tilde{\rho}_{I}\left(\Omega_{n+1}\right)=\left[\mathscr{E}+\left(-i L_{I}\left(\Omega_{n}\right)\right) \Delta t+\frac{1}{2}\left(-i L_{I}\left(\Omega_{n}\right)\right)^{2} \Delta t^{2}\right] \tilde{\rho}_{I}\left(\Omega_{n}\right)
$$

$$
\begin{aligned}
\tilde{X}_{n+1}^{k}= & \tilde{X}_{n}^{k}+a^{k}\left(\tilde{X}_{n}\right) \Delta t+1 / 2 G^{0} a^{k}\left(\tilde{X}_{n}\right) \Delta t^{2} \\
& +\sum_{j=1}^{m}\left\{b^{k, j}\left(\tilde{X}_{n}\right) \Delta W^{j}+G^{0} b^{k, j}\left(\tilde{X}_{n}\right) I_{(0, j)}\right. \\
& \left.+G^{j} a^{k}\left(\tilde{X}_{n}\right) I_{(j, 0)}\right\}+\sum_{j_{1}, j_{2}=1}^{m} G^{j_{1}} b^{k, j_{2}}\left(\tilde{X}_{n}\right) I_{\left(j_{1}, j_{2}\right)},
\end{aligned}
$$

where the stochastic integrals $\Delta W^{j} \propto(D \Delta t)^{1 / 2}$ and $I_{\left(j_{1}, j_{2}\right)} \propto(D \Delta t)^{3 / 2}$ as well as the operators $G^{i}$ are discussed further in Appendix A. Setting up this scheme can be straightforward provided eqn (8) is provided in Itô format. ${ }^{25}$ Note that no $G^{j}$ operator is needed in eqn (15) since the SLEL form has only a parametric dependence on the stochastic process $X_{t}$ (see Appendix A).

1.2.1 Remark 1: short time exponential propagator. A very common approach in the literature is to use some form of exponential short time propagator. This is true for the SLEL ${ }^{5}$ as well as the time-dependent Schrödinger equation based formulations. ${ }^{14} \mathrm{~A}$ short time exponential propagator can be written as ${ }^{7,8}$

$$
\tilde{\rho}_{I}\left(\Omega_{n+1}\right)=\exp \left[-i L_{I}\left(\Omega_{n}\right) \Delta t\right] \tilde{\rho}_{I}\left(\Omega_{n}\right) .
$$

Expanding the short time propagator to second order in the timestep gives

$$
\left[\mathscr{E}+\left(-i L_{I}\left(\Omega_{n}\right)\right) \Delta t+\frac{1}{2}\left(-i L_{I}\left(\Omega_{n}\right)\right)^{2} \Delta t^{2}\right] \tilde{\rho}_{I}\left(\Omega_{n}\right) .
$$

Thus we note that the short time exponential scheme is consistent with an explicit scheme with $\beta=2$ [cf. eqn (15)], provided for the underlying diffusion process also have $\beta=2$. As an aside, we would like to point out that explicit schemes with $\beta=2$ for diffusion process [ $c f$. eqn (16)] in combination with an exponential propagator can provide significant efficiency gains when very long trajectories are needed. ${ }^{7}$

1.2.2 Remark 2: conserved norm. Given that the density matrix is a Hermitian operator it follows that the ensemble value as well as the trajectory value of the norm has to be conserved. To see this, consider a molecular dynamics realization $\Omega_{s}$ with $s \in\left[\begin{array}{ll}0 & T\end{array}\right]$, and the formal solution of eqn (1) at time $T$ and its Hermitian conjugate given below

$$
\begin{gathered}
\rho\left(\Omega_{T}\right)=T_{+} \exp \left(-i \int_{0}^{T} \mathscr{L}\left(\Omega_{s}\right) \mathrm{d} s\right) \rho\left(\Omega_{0}\right), \\
\rho^{\dagger}\left(\Omega_{T}\right)=\rho^{\dagger}\left(\Omega_{0}\right) T_{-} \exp \left(i \int_{0}^{T} \mathscr{L}\left(\Omega_{s}\right) \mathrm{d} s\right),
\end{gathered}
$$

where $T_{ \pm}$is a time ordering operator and we note that $\mathscr{L}$ is Hermitian. We can consider a temporal discretisation $\Omega_{t_{0}}, \Omega_{t_{1}}, \ldots, \Omega_{t_{N}}$, where $t_{N}=T$ and $t_{i}-t_{i-1}=\Delta t$ for eqn (18) and (19) and compute the norm at time $T$ as follows:

$$
\begin{aligned}
\rho^{\dagger}\left(\Omega_{T}\right) \rho\left(\Omega_{T}\right)= & \rho^{\dagger}\left(\Omega_{0}\right) \exp \left(i \mathscr{L}\left(\Omega_{t_{0}}\right) \Delta t\right) \exp \left(i \mathscr{L}\left(\Omega_{t_{1}}\right) \Delta t\right) \cdots \\
& \exp \left(i \mathscr{L}\left(\Omega_{t_{N}}\right) \Delta t \times \exp \left(-i \mathscr{L}\left(\Omega_{t_{N}}\right) \Delta t\right)\right) \\
& \left.\exp \left(-i \mathscr{L}\left(\Omega_{t_{N-1}}\right) \Delta t\right) \cdots \exp \left(-i \mathscr{L}\left(\Omega_{t_{0}}\right) \Delta t\right) \rho \Omega_{t_{0}}\right) \\
= & \rho^{\dagger}\left(\Omega_{t_{0}}\right) \rho\left(\Omega_{t_{0}}\right),
\end{aligned}
$$

where $\left.\exp \left(i \mathscr{L}\left(\Omega_{t_{k}}\right) \Delta t\right) \exp \left(-i \mathscr{L}\left(\Omega_{t_{k}}\right) \Delta t\right)\right)$ is the "unitary operator". Hence, at the trajectory level we have $\rho^{\dagger}\left(\Omega_{T}\right) \rho\left(\Omega_{T}\right)=$ $\rho^{\dagger}\left(\Omega_{t_{0}}\right) \rho\left(\Omega_{t_{0}}\right)$. This observation also holds for the ensemble average. If we have an efficient way of computing the exponential 
of the Liouvillian, the Hermitian property is conserved for all timestep lengths. ${ }^{5}$ However, in practice, an exponential format is generally not available for many problems. This is because the floating point operations involved in performing diagonalisation scales as $\mathcal{O}\left(M^{3}\right)$, making this approach computationally expensive for larger problems.

1.2.3 Remark 3: error estimation. Since only numerical solutions are available to problems that are of interest, we cannot strictly evaluate the error bound given in eqn (13). However, as shown first by Talay and Tubaro, ${ }^{29}$ there exists for Itô differential equations a computable error expansion such that for an order $\beta$ numerical scheme, we have

$$
\begin{aligned}
\operatorname{dev}(T, \Delta t)= & e_{\beta}(T)(\Delta t)^{\beta}+e_{\beta+1}(T)(\Delta t)^{\beta+1} \\
& +e_{\beta+2}(T)(\Delta t)^{\beta+2}+\cdots \\
& +e_{n}(T)(\Delta t)^{n}+\mathcal{O}\left((\Delta t)^{n+1}\right),
\end{aligned}
$$

where $e_{\beta}$ are real numbers not depending on the timestep $\Delta t$. Hence, in order to assess the accuracy of a numerical scheme, we may compute a reference solution at, for instance, $\Delta t / 4$ and compute the following error estimate combining eqn (13),(14),(20).

$$
\begin{aligned}
\left|\operatorname{dev}\left(\tilde{\rho}_{\mathrm{obs}}^{\Delta t}\right)\right|= & \left|\mathscr{A}\left(\tilde{\rho}_{\mathrm{obs}}^{\Delta t / 4}(T), L\right)-\mathscr{A}\left(\tilde{\rho}_{\mathrm{obs}}^{\Delta t}(T), L\right)\right| \\
= & \left|e_{\beta}(T)\left[(\Delta t / 4)^{\beta}-(\Delta t)^{\beta}\right]+\mathcal{O}\left((\Delta t)^{\beta+1}\right)\right| \\
& \leq\left|e_{\beta}(T)\left[(\Delta t / 4)^{\beta}-(\Delta t)^{\beta}\right]\right| \leq C_{\mathrm{obs}}^{T}[\Delta t]^{\beta}
\end{aligned}
$$

This enables a mathematically rigorous verification of convergence. In the limit $\Delta t \rightarrow 0$, the solution approaches the exact solution, provided the numerical calculations are not corrupted by satistical and floating point errors.

\section{Implicit weak Itô-Taylor scheme}

Implicit schemes are widely used to solve ordinary differential equations and address numerical stability issues. In the case of stochastic differential equations similar methods can be constructed. However, the steps involved can be more complicated. ${ }^{30}$ A linear deterministic ordinary differential equation is called stiff if there exists eigenvalues whose real-parts satisfy the following inequality:

$$
\operatorname{Re}\left(\lambda_{\max }\right) \gg \operatorname{Re}\left(\lambda_{\min }\right) .
$$

In stiff problems, there exist a wide separation of time scales which imposes severe restrictions on the allowable time-step. The stiffness of stochastic differential equations can be characterised using asymptotic Lyapunov exponents. ${ }^{20}$ The numerical schemes used in previous works have generally been found to require $\Delta t<1 / \omega_{0} \rightarrow \Delta t \ll 1 / \omega_{0}{ }^{7,8}$ if a fast rate $\omega_{0}$ is explicitly present in the simulation. For instance, when a high-field approximation is valid, the numerical problem is simplified with $\omega_{0}$ effectively removed in the interaction picture [cf. eqn (6)], and for such cases a simpler explicit scheme may be the best way forward.

In what follows, we investigate if implicit numerical schemes can be employed to achieve reductions in the computational cost associated with the requirement of using short time-steps in problems with a fast rate. A family $(\alpha \in[0,1], \mu \in[0,1])$ of $\beta=1$ implicit schemes is given by by ${ }^{20}$

$$
\begin{aligned}
\tilde{\rho}_{I}\left(\Omega_{n+1}\right)= & \tilde{\rho}_{I}\left(\Omega_{n}\right)-i\left\{\alpha L_{I}\left(\Omega_{n+1}\right) \tilde{\rho}_{I}\left(\Omega_{n+1}\right)\right. \\
& \left.+(1-\alpha) L_{I}\left(\Omega_{n}\right) \tilde{\rho}_{I}\left(\Omega_{n}\right)\right\} \Delta t
\end{aligned}
$$

and

$$
\begin{aligned}
\tilde{X}_{t}^{k}= & \tilde{X}_{t}^{k}+\left\{\alpha a_{\mu}^{k}\left(\tilde{X}_{n+1}\right)+(1-\alpha) a_{\mu}^{k}\left(\tilde{X}_{n}\right)\right\} \Delta t \\
& +\sum_{j=1}^{m}\left\{\mu b^{k, j}\left(\tilde{X}_{n+1}\right)+(1-\mu) b^{k, j}\left(\tilde{X}_{n}\right)\right\} \Delta W^{j},
\end{aligned}
$$

where

$$
a_{\mu}^{k}=a^{k}-\mu \sum_{j=1}^{m} G^{j} b^{k, j}
$$

and the operators $G^{j}$ are listed in Appendix A.

It is worth noting that the computational complexity of implicit schemes at each time-step is significantly higher than the explicit $\beta=1$ scheme outlined earlier in eqn (10) and (11). Henceforth in this paper, we focus on two implicit schemes and denote them compactly by I1: $(\alpha=1, \mu=0)$ and I2: $(\alpha=1 / 2, \mu=0)$. The equations associated with the implicit scheme $\mathbf{I} 1$ are given below.

$$
\begin{aligned}
\tilde{\rho}_{I}\left(\Omega_{n+1}\right) & =\tilde{\rho}_{I}\left(\Omega_{n}\right)-i L_{I}\left(\Omega_{n+1}\right) \tilde{\rho}_{I}\left(\Omega_{n+1}\right) \Delta t, \\
\tilde{X}_{n+1}^{k} & =\tilde{X}_{n}^{k}+a^{k}\left(\tilde{X}_{n+1}\right) \Delta t+\sum_{j=1}^{m} b^{k, j}\left(\tilde{X}_{n}\right) \Delta W^{j} .
\end{aligned}
$$

The equations associated with the implicit scheme $\mathbf{I} 2$ can be written as follows:

$$
\begin{array}{r}
\tilde{\rho}_{I}\left(\Omega_{n+1}\right)=\tilde{\rho}_{I}\left(\Omega_{n}\right)-i \frac{1}{2}\left\{L_{I}\left(\Omega_{n+1}\right) \tilde{\rho}_{I}\left(\Omega_{n+1}\right)+L_{I}\left(\Omega_{n}\right) \tilde{\rho}_{I}\left(\Omega_{n}\right)\right\} \Delta t \\
(27) \\
\tilde{X}_{n+1}^{k}=\tilde{X}_{n}^{k}+\frac{1}{2}\left\{a^{k}\left(\tilde{X}_{n+1}\right)+a^{k}\left(\tilde{X}_{n}\right)\right\} \Delta t+\sum_{j=1}^{m} b^{k, j}\left(\tilde{X}_{n}\right) \Delta W^{j} .
\end{array}
$$

For the implicit scheme I2, we note that $L_{I}\left(\Omega_{n+1}\right) \tilde{\rho}_{I}\left(\Omega_{n+1}\right) \approx$ $L_{I}\left(\Omega_{n}\right)\left[\tilde{\rho}_{I}\left(\Omega_{n}\right)-i L_{I}\left(\Omega_{n}\right) \tilde{\rho}_{I}\left(\Omega_{n}\right) \Delta t\right]+\mathcal{O}\left((\Delta t)^{2}\right)$ and inserting this in eqn (27) we reccover eqn (15). Hence, even though the general original format leading to $\mathbf{I} 2$ is derived as an implicit scheme for stochastic differential equations with $\beta=1$, in the present problem, the SLEL part of the equation effectively has $\beta=2$. However, the general overall accuracy of $\mathbf{I} 2$ in terms of eqn (13) is $\beta=1$ because of the last term in eqn (28). However, depending on the application, it may be possible to formulate an implicit form of eqn (28) with $\beta=2$ at low numerical cost. ${ }^{20}$ The overall value of $\beta$ for the time-stepping scheme is important if we wish to employ extrapolation methods based on $\mathbf{I} 2 .^{26}$

In our discussion so far, we have focused on a general form of the stochastic molecular dynamics equation. However, if molecular dynamics is obtained using an atomistic MD simulation, it is reasonable to assume that the molecular dynamics trajectories are given to a discretisation error smaller than $C_{\text {obs }}^{T} \Delta t^{\beta}$. On a similar note, if some relatively slow and simple form Brownian dynamics are of interest then it may be convenient to generate these separately with a sufficiently small time step and to consider these as exact with respect to the discretisation error. 
If this the case, then the overall error of $\mathbf{I} 2$ is thus $\beta=2$. We consider now molecular dynamics given with sufficiently small discretisation error and consider only the SLEL part.

It is worth noting that for the case of the $\mathbf{I} 1$ scheme, we have to solve the following linear (and possibly nonlinear) equation at each time-step:

$$
\begin{gathered}
\tilde{\rho}_{I}\left(\Omega_{n+1}\right)=\left[\mathscr{E}+i L_{I}\left(\Omega_{n+1}\right) \Delta t\right]^{-1} \tilde{\rho}_{I}\left(\Omega_{n}\right) \\
0=f\left(\tilde{X}_{n+1}\right)=\tilde{X}_{n+1}^{k}-\tilde{X}_{n}^{k}+a^{k}\left(\tilde{X}_{n+1}\right) \Delta t+\sum_{j=1}^{m} b^{k, j}\left(\tilde{X}_{n}\right) \Delta W^{j}
\end{gathered}
$$

For scheme I2, the following linear algebraic system of equations need to be solved at each timestep

$$
\begin{gathered}
\underbrace{\left[\mathscr{E}+i / 2 L_{I}\left(\Omega_{n+1}\right) \Delta t\right]}_{K} \underbrace{\tilde{\rho}_{I}\left(\Omega_{n+1}\right)}_{x} \\
=\underbrace{\left[\mathscr{E}-i / 2 L_{I}\left(\Omega_{n}\right) \Delta t\right] \tilde{\rho}_{I}\left(\Omega_{n}\right)}_{b},
\end{gathered}
$$

and thus ensuring equality between an approximate half conjugate step with half forward step. To evaluate the implicit SLEL (I1, I2) [cf. eqn (29),(31)], we need to solve a possibly high-dimensional but sparse linear algebraic system of equations at each time-step. The matrix $L_{I}\left(\Omega_{n+1}\right)$ is Hermitian, however the matrices in eqn (29),(31) are complex symmetric. The problem of solving the linear equation for a high dimensional case is ideally suited for an iterative Krylov subspace method such as GMRES ${ }^{31}$ for which efficient numerical implementations are available in the publicdomain. In the present work, we used the PETSc library. ${ }^{32-34}$

\subsection{Computational cost and memory requirements}

We now summarise the computational cost associated with solving the SLEL using the schemes outlined earlier (E1, E2, I1 and I2). The memory requirements and floating point operations for all the schemes are summarized in Table 1.

We refer to Appendix B and eqn (3) for a detailed example of the Liouvillian and the notation used. We note that with Zeeman interaction and three nuclear spins there are 20 submatrices with non-diagonal elements. The computationally most expensive step in scheme I1 involves solving the linear system of equations ( $c f$. eqn (29)). The scheme $\mathbf{I} 2$ also involves solving a linear system of equations (eqn (31)) and an additional matrix-vector multiplication. However, as shown later via numerical studies, for the class

Table 1 The first row gives basic memory requirements for E1, E2, I1 and $\mathbf{I} 2$. The subscript $i$ runs over five submatrices for each interaction included in spin Hamlitionian [cf. eqn (3)], each of size $L=M \times M$. I1 and $\mathbf{I} 2$ involves the assembly of an additional matrix of size $L$ and if a preconditioner is used, then an additional matrix of size $L$ needs to be stored hence the (3). In the second row, scalar function times matrix corresponds to the assemble cost of $L$, two at each timestep for $\mathbf{I} 2$. The third row summarizes the number of matrix vector multiplications. The fourth row shows the linear equation to be solved for I 1 and I 2

\begin{tabular}{lllll}
\hline & E1 & E2 & I1 & I2 \\
\hline Storage & 1 & 1 & $2(3)$ & $2(3)$ \\
$\rho_{M}+\sum_{i} A(i)_{M \times M}$ & 1 & & & \\
Operations & 1 & 1 & 1 & 2 \\
$\sum_{i} F\left(\Omega_{t}\right) A(i)_{M \times M}$ & 1 & 3 & 0 & 1 \\
$\rho_{M}+L_{M \times M} \rho_{M}$ & 0 & 0 & 1 & 1 \\
Solve $K_{M \times M} x=b$ & 0 & & & \\
\hline
\end{tabular}

of problems considered in the present study, implicit schemes are more preferable from an accuracy and computational efficiency point of view. The present implementation of implicit schemes using the PETSc library scales very well to large-scale matrices since distributed memory matrix storage schemes can be exploited $^{34}$ (this feature was not needed for the problems studied here, the larger problem required only around $800 \mathrm{MB}$ memory).

\section{Results and discussion}

We follow up previous works ${ }^{8}$ and $^{7}$ and explore the performance of implicit and explicit numerical schemes. Extensive numerical studies are carried out to address the following questions: (i) what is the maximum allowable timestep for various schemes? (ii) how does various schemes perform in different dynamical regimes? and (iii) which schemes ensure conservation of the norm of the density operator. The first question is relevant to assess the possibility for further efficiency improvements by extrapolation. The second question is relevant to identify experimental conditions (for instance temperature solvent viscosity and spectrometer frequency) making experiments more accessible to interpretation by numerically solving the SLEL. The third question is critically important to validate numerical schemes for problems where we solve for an Hermitian density matrix. We consider these details carefully for an transition metal EPR problem and then coment on the fluorescence dipolar interaction problem studied in ref. 8 .

\subsection{EPR for a transition metal complex}

To the best of the authors' knowledge, an EPR observable for a $\mathrm{Cu}(\mathrm{II})$ transition metal complex has never been computed using the SLEL formalism. EPR on Cu(II) complexes may provide detailed molecular information of the transition metal center, ${ }^{35}$ information that is made available with a rigorous solution of the SLEL. Currently, there is no rigorous approach available that can interpret a room temperature EPR spectra of a $\mathrm{Cu}$-complex with several ligands. Thus working towards an efficient solver for the SLEL capable of efficient $\mathrm{Cu}$-complex calculations is a relevant problem and suitable as a test case. We are interested in the temporal discretization error in the SLEL solution and for this reason we implement a simple one dimensional Brownian dynamics process with

$$
\overline{\Omega_{t}^{2}}=2 D\left(t-t_{0}\right) \text { and } \Omega_{t_{k}}=\sum_{i=0}^{k} \sqrt{2 D \Delta t} \xi_{i},
$$

where $\Omega_{t}=\left\{0, \beta_{t}, 0\right\}$ and $\xi_{i}$ are $N(0,1)$ uncorrelated Gaussian random variables. The spin Hamiltonian elements can be directly evaluated (see Appendix B and note that $\beta_{t}$ is the lab to principal reference frame polar angle), hence eliminating the discretisation error from the diffusion process used to represent molecular dynamics. In this model, the fluctuations in the spin Hamiltonian components $F_{\mu, \eta}^{(2, m)}$ (see Appendix B) are characterised by $\overline{F\left(\Omega_{0}\right) F\left(\Omega_{t}\right)}=F^{2} \exp \left(-t / \tau_{c}\right)$. The relevant $L$ matrices (cf. eqn (3)) are set up using a Zeeman superoperator basis and is conveniently done with mPackages subroutines ${ }^{36}$ for different matrix sizes and magnetic resonance problems.

We consider the orders of magnitude larger anisotropy seen in transition metal complex magnetic tensors with typical $\mathrm{Cu}$ (II) values in Table 3 , compared to nitroxide spinprobe type 
Table 2 Cases with $\omega_{0} / \sqrt{\Delta H^{2}}=10$

\begin{tabular}{lll}
\hline Case & $\sqrt{\Delta H_{\max }^{2}} \tau_{c}$ & $\omega_{0} \tau_{c}$ \\
\hline I & 0.1 & 1 \\
II & 1 & 10 \\
III & 10 & 100 \\
\hline
\end{tabular}

of values. For the X-band experimental conditions considered here, it is the field dependent part of Hamiltonian that is the dominating relaxation mechanism (denoted as $\sqrt{\Delta H_{\max }^{2}} \tau_{c}$ ). The three dynamical regimes listed in Table 2 are explored using a $36 \times 36$ Liouville matrix for a $S=1 / 2, I=1$ system. To explore a high dimensional system, we consider the $5184 \times 5184$ Liouville matrix for $S=1 / 2, I_{1}=3 / 2, \mathrm{I}_{2}=1, I_{3}=1$, which corresponds to a square planar copper complex with two nitrogen ligands. Typical experimental observables for these systems are computed as an ensemble average over a complete representation of different initial condition $\Omega_{0}$. However, in the present study, since our focus is on discretization error, we restrict our numerical study to one initial condition that has large discretisation and statistical errors $\left(\Omega_{0}=\left\{0,73^{\circ}, 0\right\}\right)$.

For the implicit schemes, we find that the GMRES algorithm used for solving the linear algebraic equations at every timestep converges without a preconditioner to sufficiently low tolerance $\left(10^{-8}\right)$ with up to 6 iterations. The need for very few iterations is primarily due to the good initial guess provided to GMRES in the previous timestep for I1 (and the half Euler step that is the initial guess for I2; see eqn (31)). The tolerance determining the maximum number of iterations used while solving the linear equations is kept high without affecting the EPR observable. The amplitude of the observable for the larger system over a $6 \mathrm{~ns}$ time window computed using the numerical schemes $\mathbf{I} 2$ and $\mathbf{E} 2$ are shown in the left and right panel of Fig. 1, respectively. It is observed that the observable computed with a large timestep shows a small deviation from the reference calculation.

To quantify the deviation, we compute the observable using different timesteps and compute $\operatorname{dev}(T, \Delta t)$ [cf. eqn (13),(21)], with a reference calculation at $\Delta t=0.0025 \mathrm{~ns}$ ( or $\left.2 \pi \omega_{0} \Delta t=0.025\right)$ and calculate the root mean square value of $\operatorname{dev}(T, \Delta t)$ over the five time points in Fig. 1 . We note that the $36 \times 36$ observable decays up to 10 times slower making longer trajectories relevant. Thus, to test conservation of the norm of the density operator we evaluate $\operatorname{dev}(T, \Delta t)$ at $T=60$ ns. Fig. 2 shows $\operatorname{dev}(T, \Delta t)$ for norm $\left.\overline{\left(\rho^{\dagger}\left(\Omega_{T}\right) \rho\left(\Omega_{T}\right)\right.}\right)$ and $\overline{\rho_{\text {obs }}}$, the former quantity versus timestep and the latter quantity versus timestep and computational time.

The following observations can be drawn from the results obtained.

1. The deviation in the norm $\left(\overline{\rho^{\dagger}\left(\boldsymbol{\Omega}_{T}\right) \rho\left(\boldsymbol{\Omega}_{T}\right)}\right)$ follow $\beta=2$ for $\mathbf{I} 2$ and $\beta=1$ for $\mathbf{I} 1$ (small timestep) and closer to $\beta=3$ with a large prefactor for E2. Since the norm is critically

Table 3 Magnetic tensors for ${ }^{63} \mathrm{Cu}$ (II) with two equivalent nitrogen $(j=2,3)$, units are in gauss where applicable

\begin{tabular}{llllllll}
\hline$g_{n}$ & $g_{\perp}$ & $g_{\|}$ & $B_{0}$ & $A_{1 \perp}$ & $A_{1 \|}$ & $A_{j \perp}^{N}$ & $A_{j \|}^{N}$ \\
\hline 1.484 & 2.05 & 2.18 & 3400 & 18.4 & 190.4 & 17.1 & 12.9 \\
\hline
\end{tabular}

important for the problem we only proceed to calculate the observable (row two and three) for $\Delta t<\Delta t_{\max }$. The norm is a conserved quantity and as such it should not have any statistical variability. We estimated the standard deviation and found it to be lowest for $\mathbf{I} 2$, with values in the range of $10^{-12}-10^{-7}$. In contrast, for $\mathbf{E} 2$ with timestep $\sim 0.75$, the standard deviation is $10^{+3}$, which means that this scheme fails at moderate size timesteps. Such failures are not observed for the implicit schemes $\mathbf{I} 1$ and $\mathbf{I} 2$.

2. From the second row of Fig. 2, it can be seen that, in general, the observable computed using $\mathbf{I} 2$ follow $\beta=2$ for $\Delta t_{\max }=1 / 2 \pi \omega_{0}$. However, $\Delta t_{\max }$ is higher for the dynamical regime of case II.

3. It can be noted from the bottom row of Fig. 2 that at longer computational time, all the schemes give similar results within statistical error. However if a slightly larger error is acceptable, then the implicit scheme $\mathbf{I} 2$ can be used with an order of magnitude reduction in computational time.

The norm of the density operator is a basic property that needs particular attention in order to ensure that the calculated observable is meaningful. We illustrate with Fig. 3 the behaviour of $\|\bar{\rho}\|$ for the schemes $\mathbf{I} 2$ and E2 using two different timesteps. The deviation in the norm is exceptionally small for $\mathbf{I} 2$ and remains constant over time, whereas there is a linear growth in error for E2. This suggests that $\mathbf{I} 2$ is an attractive numerical scheme, particularly for problems where the observable under study is slowly relaxing (e.g., spin-lattice relaxation which is a $T_{1}$ process).

For the larger spin system shown in Fig. 4, the deviation in the observable is displayed as a function of the time step and the computational time. It can be noted from the left panel of this figure that the trends observed for this case are similar to those observed earlier for the smaller system with $\Delta t_{\max }=1 / 2 \pi \omega_{0}$. E2 gives similar results as $\mathbf{I} 2$ for short timesteps. The computational time is somewhat less for $\mathbf{E} 2$ as seen in the right panel. However, the implicit scheme $\mathbf{I} 2$ is the method of choice, at least for $\mathrm{Cu}(\mathrm{II})$ complexes, since this scheme conserves the norm of the density operator and allows for all types of observables to be computed. From the numerical studies, it is found that similar computational effort is needed in a wide range of dynamical regimes.

\subsection{Fluorophore dipolar interaction}

We consider the same fluorescence problem as in ref. 8 since it is a low dimensional problem and in addition explicit schemes where found to be costly to apply due to the presence of a large relaxation rate. Furthermore, for this problem the exponential propagator eqn (17) can be evaluated very cheaply. ${ }^{8}$ The Liouvillian for dipole pair $A-D$ has the form

$$
-i L\left(\Omega_{t}\right)=\left(\begin{array}{cccc}
-1 / \tau_{A} & 0 & i H\left(\Omega_{t}\right) & -i H\left(\Omega_{t}\right) \\
0 & -1 / \tau_{D} & -i H\left(\Omega_{t}\right) & i H\left(\Omega_{t}\right) \\
i H\left(\Omega_{t}\right) & -i H\left(\Omega_{t}\right) & C & 0 \\
-i H\left(\Omega_{t}\right) & i H\left(\Omega_{t}\right) & 0 & C^{*}
\end{array}\right),
$$

where the matrix elements $1 / \tau_{A}$ and $1 / \tau_{D}$ refer to individual fluorescence relaxation rates and $C=i \delta-1 / T_{2 A}-1 / T_{2 D}$ 

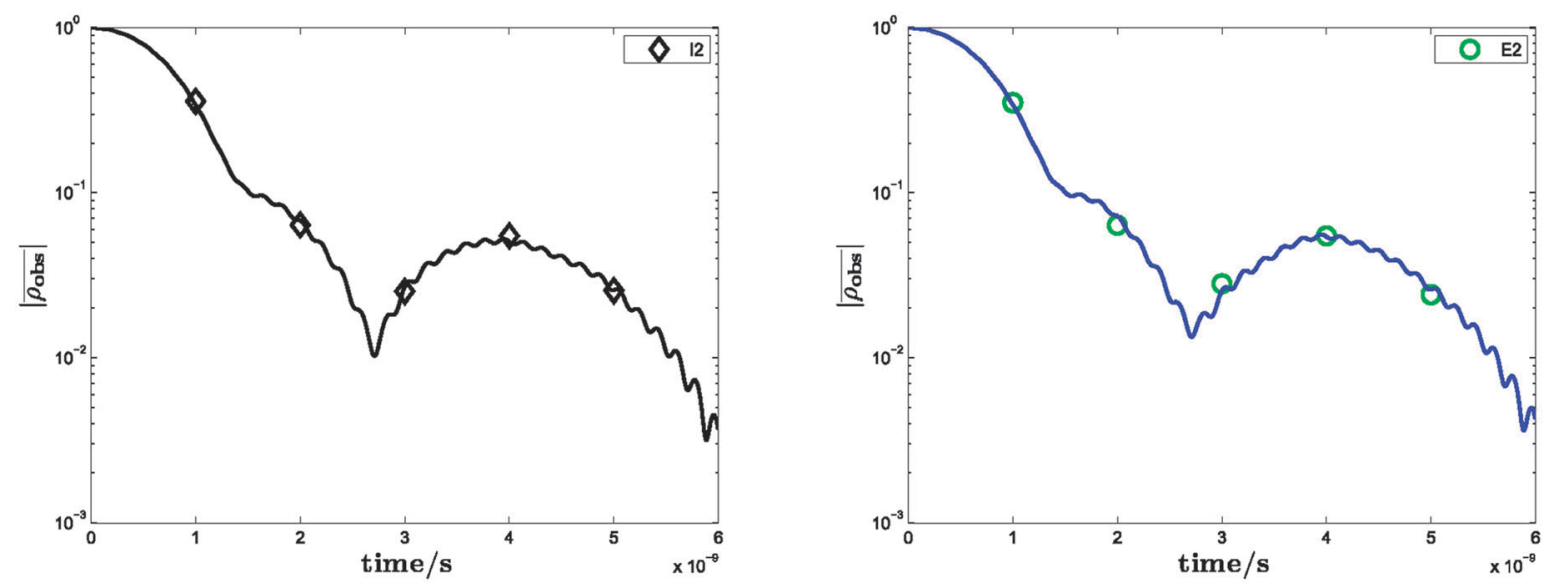

Fig. 1 Semilog plots of $\left|\overline{\rho_{\text {obs }}}\right|$ as a function of time for $5184 \times 5184$ Liouville matrix for the numerical schemes $\mathbf{I} 2(\diamond)$ and $\mathbf{E} 2(\bigcirc)\left(\right.$ with $\left.2 \pi \omega_{0} \Delta t=0.5\right)$ in left and right panel, respectively (five representative points). Solid lines are simulation results obtained using $2 \pi \omega_{0} \Delta t=0.025$.

I
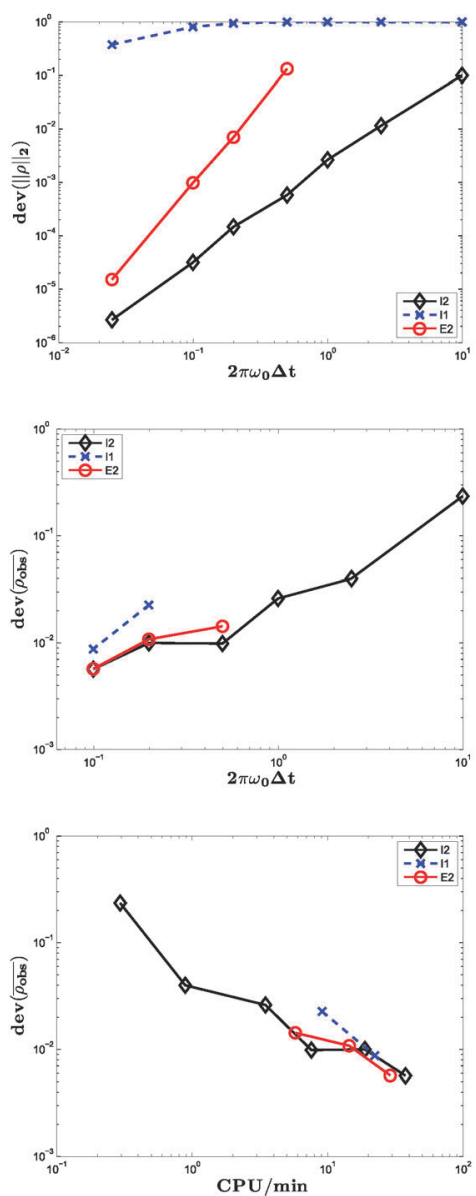

II
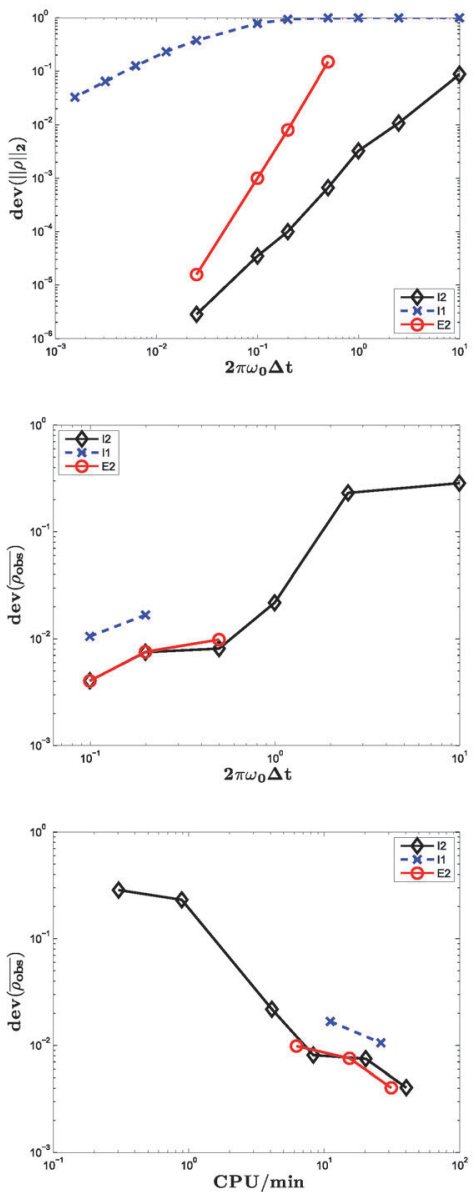

III
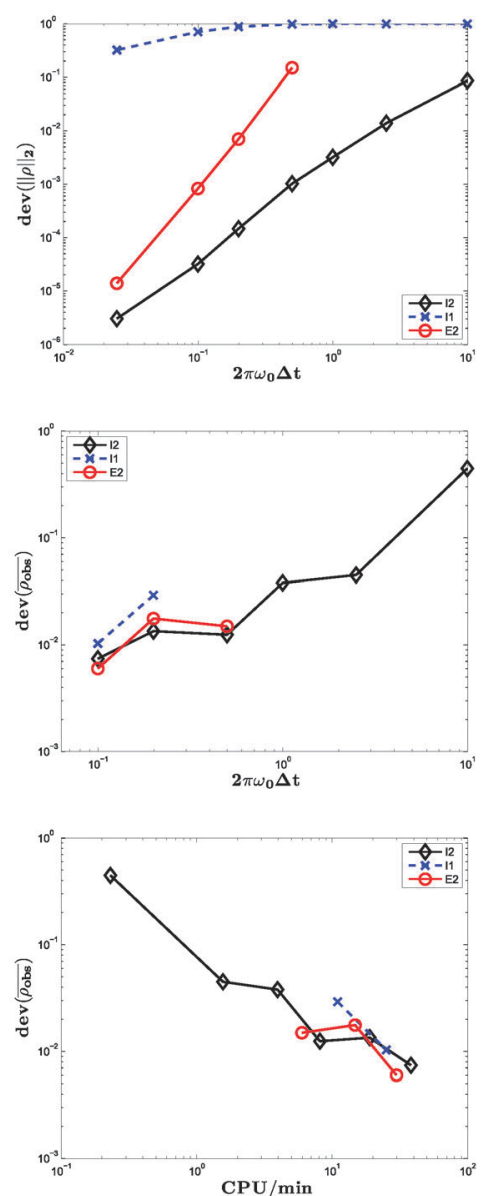

Fig. 2 For Liouville matrix $36 \times 36$, top row $\mid \operatorname{dev}\left(\| \rho^{\Delta t}\left(\Omega_{T} \|\right) \mid T=60\right.$ ns [cf. eqn (21)], row two and three $\sqrt{1 / 5 \sum_{i=1}^{5} \operatorname{dev}\left(\rho_{\text {obs }}^{\Delta t}\left(\Omega_{T_{i}}\right)\right)^{2}}$ versus time step and single processor computational time respectively (for selection of $T_{i}$ see text). Row two and three requires a reference calculation that was computed with $2 \pi \omega_{0} \Delta t=0.025$. In top row the trajectory number is $L=50$ and for the two bottom rows the calculation proceed until estimated statistical error $\log _{10}\left(\sqrt{\sigma^{2}(r m s(o b s)) / L}\right) \leq-2.3$ over the five points.

accounts for spectral overlap and widths of individual absorption peaks of $A$ and $D$, respectively, and we denote $1 / T_{2}=1 / T_{2 A}+1 / T_{2 D}$. The dipolar Hamiltonian is dependent on a stochastic process in time $\left(\Omega_{t}\right)$ caused by relative angular displacements of dipole $A$ and $D$. We refer to ${ }^{8}$ for further discussion of the problem and to ref. 37 for the first derivation 

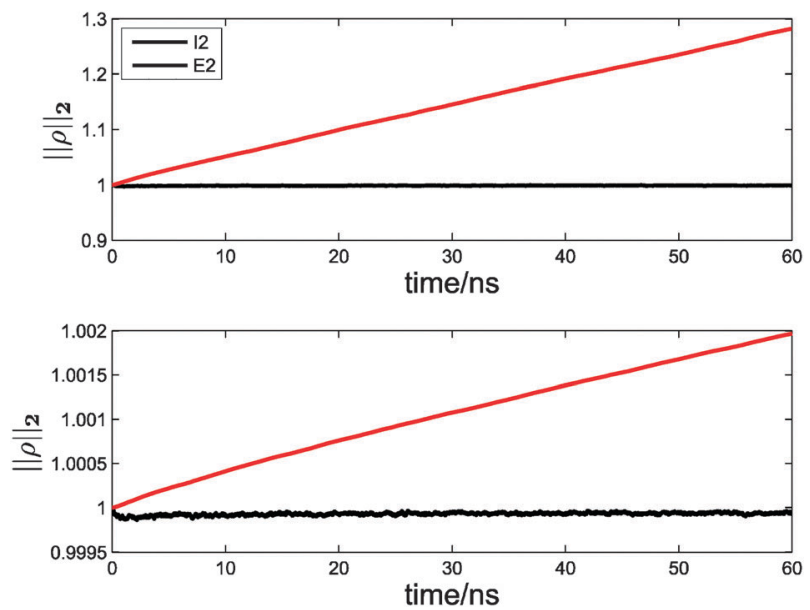

Fig. 3 For $36 \times 36$ Liouville matrix, dynamical regime I, $\|\rho\|_{2}$ versus time, timestep $2 \pi \omega_{0}=0.5$ and $2 \pi \omega_{0}=0.1$ in upper and lower panel respectively.

of the Liouvillian starting from the complete $M=16$ basis. The relevant basis set considered here is

$\left\{\rho_{1}, \rho_{2}, \ldots, \rho_{4}\right\}=\{\mid 00)_{A}\left(\left.11\right|_{D}, \mid 11\right)_{A}\left(\left.00\right|_{D}, \mid 01\right)_{A}\left(\left.10\right|_{D}, \mid 10\right)_{A}\left(\left.01\right|_{D}\right\}$,

and we compute the excitation probability of initially excited fluorophore at later time $T$ by $\overline{\rho_{1}\left(\Omega_{0}\right)^{\dagger} \rho_{1}\left(\Omega_{T}\right)}$. We are primarily interested in the numerical properties with focus on the SLEL part. Hence, we generate one set of trajectories of $\Omega_{t}$ from pairs of Brownian spherical reorientation processes ${ }^{25,27}$ using a small time step and the same set of trajectory is used when computing the SLEL observable at different timesteps. In this approach it is straight forward to find an appropriate timestep that makes the discretisation error in molecular dynamics $\Omega_{t}$ negligible. With $\tau_{c}$ as the characteristic correlation time of $H\left(\Omega_{t}\right)$, we choose a physically meaningful diffusion constant and dipolar coupling strength ${ }^{38}$ and $T_{2}$ such that $\sqrt{\Delta H^{2}} \tau_{c}=325$, $\left(1 / T_{2}\right) \tau_{c}=10^{5}$, with $\delta=0$ (similar results are also observed with non-zero values of $\delta$ in this parameter regime). This parameter set corresponds to a weak coupling regime where the dimensionality of eqn (32) can be reduced as shown in ref. 8. However, since $\left(\Delta H^{2} T_{2}\right) \tau_{c}=1.2$, this problem does not permit a perturbative treatment and hence a simulation method becomes necessary. Finally, we choose $\tau_{A}=\tau_{D}$ and $\Delta H^{2} T_{2} \ll 1 / \tau_{A}$, so that the fluorescence relaxation rates have a negligible influence on the observable.

We compute the deviation (dev) [cf. eqn (21)] in the observable at time $t_{N} \approx \tau_{c}$ (the reference simulation result calculated using a very small timestep $\Delta t / T_{2}=0.068$ ). We simply note from Fig. 5 that $\mathbf{I} 2$ is particularly advantageous for this parameter set since we can use a $\sim 10^{4}$ times larger $\Delta t_{\text {max }}$ compared to $\mathbf{E} 1$ and exponential propagator. The efficiency gain $(\mathrm{CPU} / \mathrm{min})$ is of similar magnitude. This finding of large efficiency gain may of course be directly valuable for some problems, however, we note that the reduced dimensional formulation in ref. 8 finds the same solution and with similar computational cost as $\mathbf{I} 2$ for this particular parameter set. Hence, in this very specific context, the gain is on the theoretical side, since $\mathbf{I} 2$ enables us to numerically verify the performance of a simplified reformulation. Nevertheless, it is worth noting that the implicit scheme $\mathbf{I} 2$ is expected to very useful for more general fluorescence problems that do not allow for such a simplified treatment. For instance, under different excitation conditions, the complete $(16 \times 16)$ Liouvillian is relevant ${ }^{37}$ and a low cost exponential propagator can be difficult to set up.

\subsection{Experimental examples}

The convergence study for the EPR observable described above was concerned with only one initial orientation and a one-dimensional Brownian process. To interpret EPR experiments with isotropic solvents, a Brownian motion on a sphere is commonly used. Furthermore, a complete representation of initial angles are required in SLEL simulation (refereed to as powder angles). To provide an example of experimental observable, we compute FID and lineshapes for typical nitroxide spin probe and square planar copper complex spin Hamiltonian parameters listed in Tables 3 and 4, respectively. Initial orientations of molecular trajectories are sampled randomly on a sphere (i.e. probability density $\propto \sin (\beta)$ for $\beta_{0}$ and uniform $[0,2 \pi]$ for $\left.\alpha_{0}\right)$. We note that extensive work have gone into

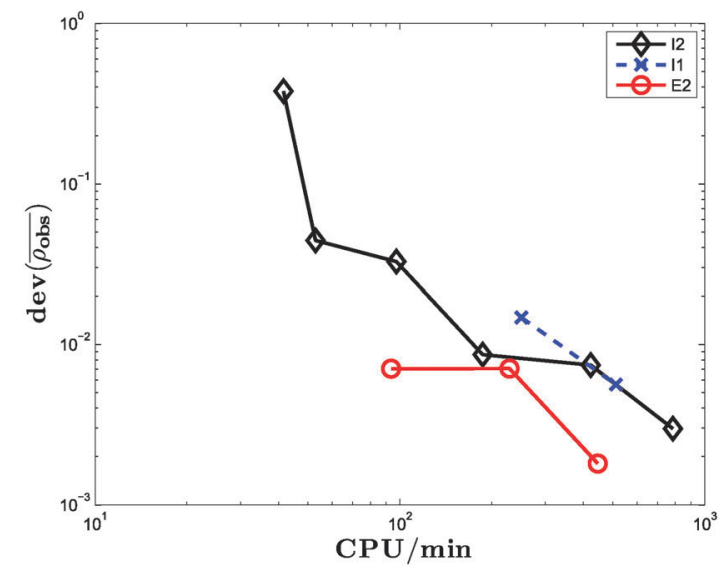

Fig. $4 \log -\log$ plots of $\operatorname{dev}\left(\rho_{\text {obs }}^{\Delta t}\right) \approx \sqrt{1 / 5 \sum_{i=1}^{5}\left(\mathscr{A}\left(\rho_{\text {obs }}^{\Delta t}\left(T_{i}\right), L\right)-\operatorname{ref}\left(T_{i}\right)\right)^{2}}$ as a function of time step and single processor computational time in left and right panel, respectively, for $5184 \times 5184$ Liouville matrix. One standard error is -2.46 in $\log$ scale. 

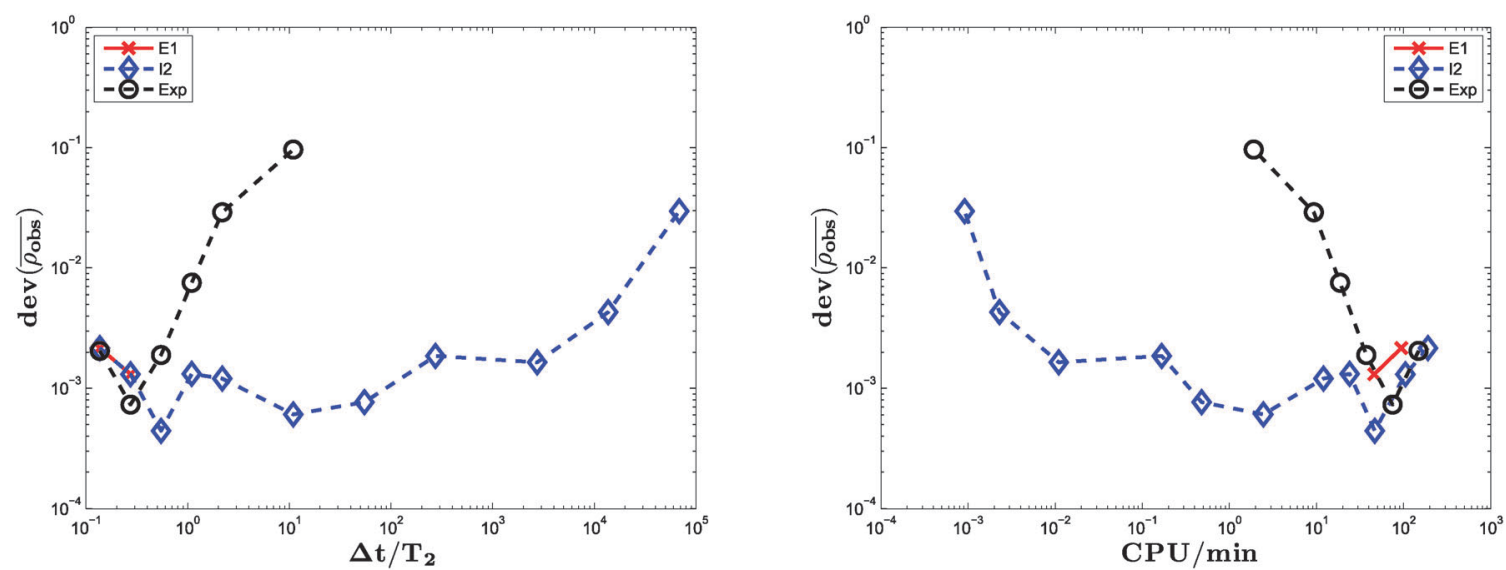

Fig. $5 \mathrm{Log}-\log$ plots of $\operatorname{dev}\left(\rho_{\mathrm{obs}}^{\Delta t}[c f\right.$. eqn (21)] vs. timestep $(\Delta t)$ and $\operatorname{dev} v s$. CPU time in left and right panel respectively. Reference calculation is computed with $\Delta t / T_{2}=0.068$ using the respective numerical schemes. Estimated standard errors are $\log _{10}\left(\sqrt{\sigma^{2} / L}\right)=-2.75$. The numerical schemes $(\mathbf{E} 1, \times),(\mathbf{I} 2, \diamond$, where linear equation is solved by direct inversion) and exponential propagator (eqn $(17), \bigcirc)$ are used.

finding efficient minimal set of powder angles in the NMR community (see for instance references at http://www.mhl. soton.ac.uk). We found for instance that a set of $\sim 100$ angles work well for the nitroxide spinprobe problem. ${ }^{39}$ However, for the almost 1000 gauss bandwidth high spin problem, small ripples remain in lineshape even for a large set 1100 of powder angles (data not shown). Thus we perform only random sampling of powder angles at this stage of code development.

In Fig. 6 we provide the amplitude of FID and absorption lineshape for nitroxide spinprobe computed with the I2 scheme computed with $36 \times 36$ and $9 \times 9$ Liouville matrix, denoted by SLEL and SLEL-reduce, respectively. The absorption lineshape is computed with eigen function expansion in the frequency domain (SLE-FP). ${ }^{40,41}$ The following observations can be made from Fig. 6 .

1. The SLEL approach based on the implicit scheme $\mathbf{I} 2$ is in excellent agreement with the SLE-FP approach that is an established method with 40 years of use on spin probe problems. ${ }^{4}$

2. Simulations conducted using the $\mathbf{I} 2$ scheme with complete (SLEL) and high-field approximated (SLEL-reduce) Liouville matrix are in good agreement. Thus it is verified numerically for the first time that the high-field approximation is valid for SLEL calculation of nitroxide spinprobe within the selected molecular dynamics model and X-band resonance frequency.

To verify the generality of above two observations, we conducted tests at various dynamical regimes within $1 \leq \omega_{0} \tau_{c} \leq 100$ (data not shown). We note in this context that the nitroxide spin probe problem with a $9 \times 9$ Liouville matrix (SLEL-reduce) may be performed more cost effectively (using longer timesteps). The Trotter splitting approach ${ }^{5,21}$ have proven to be efficient for this problem.

Using the implicit scheme $\mathbf{I} 2$ we can address problems that previously could only be tackled using a highly approximate method $^{42}$ and never within a SLEL simulation framework.

Table 4 Magnetic tensors for nitroxide spinprobe, units are in gauss where applicable

\begin{tabular}{llllll}
\hline$g_{n}$ & $g_{\perp}$ & $g_{\|}$ & $B_{0}$ & $A_{\perp}$ & $A_{\|}$ \\
\hline 0.404 & 2.0088 & 2.0027 & 3400 & 6.1 & 32.6 \\
\hline
\end{tabular}

Fig. 7 shows the amplitude of FID and first derivative X-band lineshape observable with spin Hamiltonian parameters typically seen for a square planar $\mathrm{Cu}$ (II)-complex with two nitrogen ligands. The lineshape have features seen in experiments for a fluid sample and slowly tumbling complex without the resolved ligand hyperfine splittings. (see for instance Fig. 4 in ref. 43, though spin Hamiltonian parameters in that ref have lower $g_{\|}$and higher $A_{\|}^{\mathrm{N}}$ so not quantitatively comparable with the spectra).

The computational cost for experimentally interesting cases is acceptable at around $3 \mathrm{~h}$ using $882.27 \mathrm{GHz}$ processors and the memory requirement for solving these problems is less than $1 \mathrm{~GB}$ which is not an issue for modern computers. If molecular dynamics is a costly part of the simulation, then the ergodic property of molecular dynamics may be exploited ${ }^{21}$ and trajectories can recycled when computing the powder average. $^{7}$

To summarize the experimental test problem we note that we found agreement with the eigen function expansion method (denoted SLE-FP) considering a spinprobe in solution. The spinprobe problem is solved within fractions of a second using SLE-FP,${ }^{4}$ thus a comment on usefulness of $\mathbf{I} 2$ is relevant. We note that the problem changes drastically with the large anisotropy seen for $\mathrm{Cu}(\mathrm{II})$-complex with ligands. An extension of the SLE-FP approach has been precluded for a long time, lacking a program that can handle more than one hyperfine interaction and large anisotropies in tensors while still be numerically stable. ${ }^{35,44}$ The $\mathbf{I} 2$ numerical scheme in EPR problems is expected to be particularly useful if an anisotropic rotational diffusion model is required for molecular motion. The Liouville matrix dimension in SLE-FP will in this case strictly grow to infinity since the number of eigen functions is unbounded, ${ }^{45}$ whereas $\mathbf{I} 2$ have similar cost with an anisotropic model. However, the major advantage for $\mathbf{I} 2$ is expected where diffusion limit is found unsatisfactory and Molecular Dynamics simulation can be performed. A few nano seconds of atomistic Molecular Dynamics required for a $\mathrm{Cu}$ (II)complex EPR observable can be done routinely for several problems and can be imported in $\mathbf{I} 2$ following the methodology of ref. 21 . 

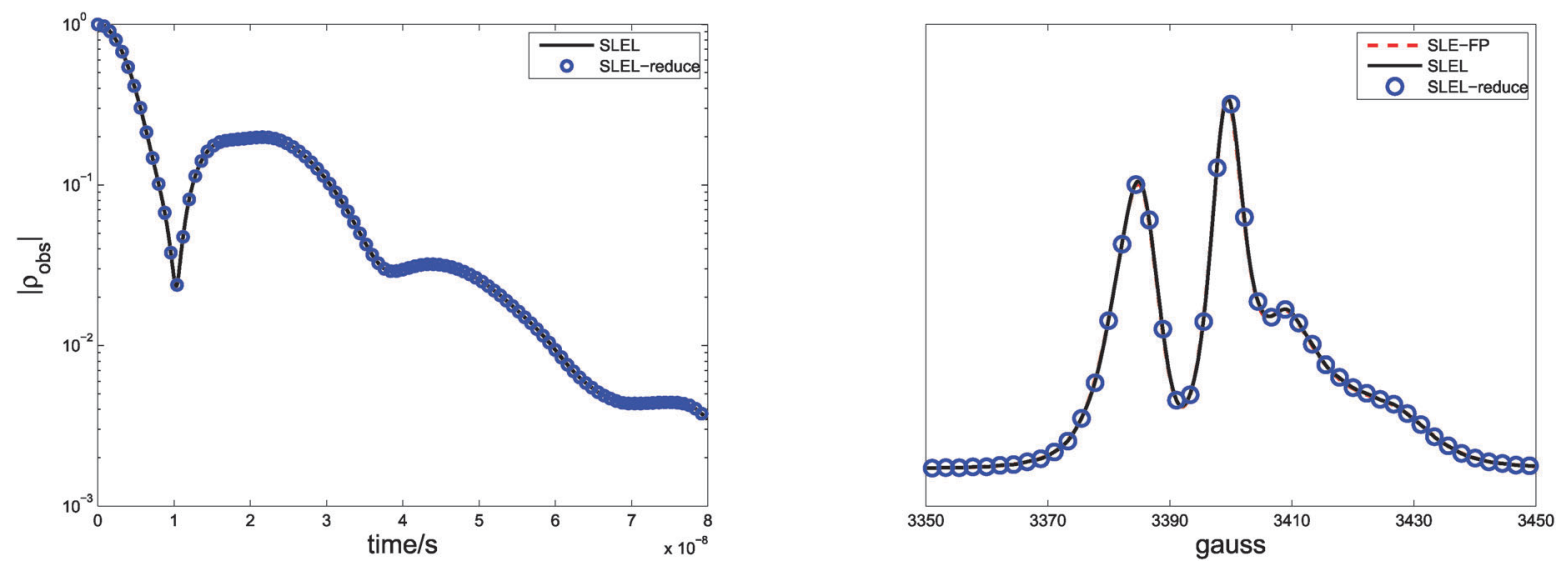

Fig. 6 Amplitude of FID $\left(\left|\overline{\rho_{o b s}}\right|\right)$ and absorption X-band EPR observable for Nitroxide spinprobe spin Hamiltonian parameters in left and right panel respectively. Frequency domain eigen function expansion (SLE-FP) and $\mathbf{I} 2$ numerical scheme is used with full $36 \times 36$ (SLEL) and high-field approximation $9 \times 9$ (SLEL-reduce) matrices respectively. Brownian motion on a sphere with diffusion constant $D=3.2 \times 10^{7} \mathrm{~s}^{-1}, \mathbf{I} 2$ timestep $2 \pi \omega_{0} \Delta t=0.35$ and $L=500000$ trajectories. Computational time is $2.5 \mathrm{~h}$ employing 88 processors.
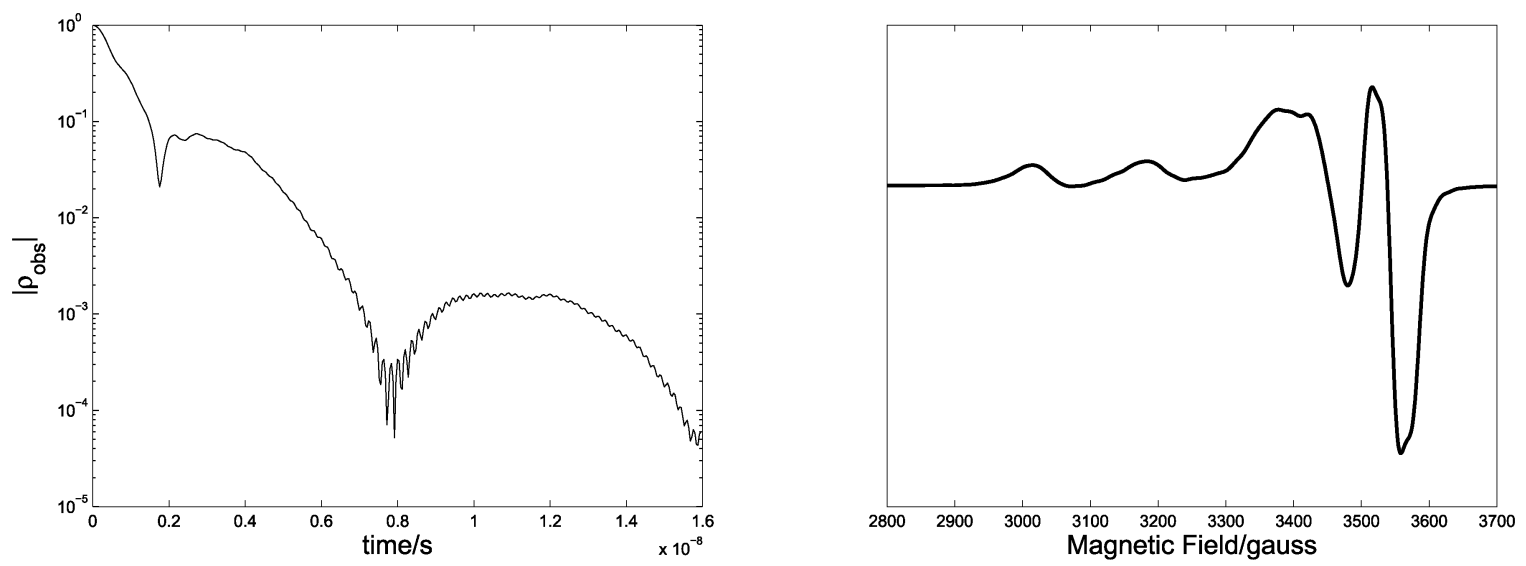

Fig. 7 Amplitude of FID $\left(\left|\overline{\rho_{\text {obs }}}\right|\right)$ and first derivative X-band EPR observable for $5184 \times 5184$ Liouville matrix in left and right panel respectively and numerical schemes I2. Magnetic tensors in Table 3. Molecular trajectories are rotational Brownian motion on sphere with diffusion constant $D=4.8 \times 10^{7} \mathrm{~s}^{-1}$ SLEL timestep is $2 \pi \omega_{0} \Delta t=0.35$ and $L=49000$ trajectories. Computational time is $2.3 \mathrm{~h}$ employing 88 processors.

\section{Conclusion}

In this work we investigated two implicit numerical schemes for SLEL relaxation studies. The performance of the proposed schemes were numerically tested on and EPR and fluorescence dipolar interaction problems and the results obtained were compared against standard explicit schemes. Implicit schemes have not been considered previously in the specific SLEL spectroscopic applications to the best of our knowledge. It is found that it is relevant to treat problems with an implicit scheme when there is a fast relaxation rate present. Furthermore, an implicit scheme is found to be cost effective since only a sparse linear system of equation needs to be solved at each timestep. For a transition metal EPR problem, the implicit scheme $\mathbf{2} 2$ allows for the complete Hamiltonian including nonsecular terms to be treated within the SLEL simulation, which is expected to be a necessity for several EPR problems. ${ }^{6}$ In addition the implicit scheme I2 provides a very good approximation, allows for a wider range of timesteps, and conserves the norm of the density operator.

It is a common feature in trajectory based methods that the statistical error is a restricting factor. However, using a robust underlying time-stepping scheme, recent developments with extrapolation on the $\mathrm{fly}^{26}$ or multilevel path simulation ${ }^{46}$ can free computational resources such that more trajectories can be computed. Variance reduction techniques can also be employed to improve efficiency. ${ }^{20}$ These are aspects that will be addressed in future work. We find that the implicit scheme $\mathbf{I} 2$ provides a very interesting candidate, opening up avenues for several new spectroscopic studies. For instance, high-dimensional spin systems where transition metals interact strongly with several ligands is a biologically relevant problem in EPR study of metalloproteins, ${ }^{47}$ that may be performed under physiological temperatures. Using the presented $\mathbf{I} 2$ scheme, it becomes possible to widen the range of problems that are within reach for a rigorous interpretation at the molecular level.

\section{A. Appendix}

In this appendix, we consider the SLEL together with a molecular dynamics process in Itô diffusion format. ${ }^{25}$ With an 
$M \times M$ dimensional Liouville matrix and $m$ dimensional diffusion $X_{t}$, we may write

$$
\begin{aligned}
\hat{X}_{t} & =\left(\begin{array}{c}
\rho_{I}\left(\Omega_{t}\right) \\
X_{t}
\end{array}\right) ; \hat{A}\left(\hat{X}_{t}\right)=\left(\begin{array}{c}
-i L_{I}\left(\Omega_{t}\right) \rho_{I}\left(\Omega_{t}\right) \\
A\left(X_{t}\right)
\end{array}\right) ; \\
\hat{B}\left(\hat{X}_{t}\right) & =\left(\begin{array}{cc}
0 & 0 \\
0 & B\left(X_{t}\right)
\end{array}\right) \hat{W}_{t}=\left(\begin{array}{c}
0 \\
\\
W_{t}
\end{array}\right)
\end{aligned}
$$

and the problem may now be restated as follows:

$$
\hat{X}_{t}=\hat{X}_{0}+\int_{0}^{t} \hat{A}\left(\hat{X}_{s}\right) d s+\int_{0}^{t} \hat{B}\left(\hat{X}_{s}\right) d \hat{W}_{s} .
$$

Given the format of eqn (A.2), we can use the weak Itô-Taylor expansion and benefit from several mathematical properties of the solution such as those listed in eqn (13) but also the existence of an error expansion and several others. ${ }^{20}$

An explicit weak scheme can be constructed from ref. 20 by defining the operators

$G^{0}=\frac{\partial}{\partial t}+\sum_{k=1}^{M+m} \hat{a}^{k}\left(X_{t}\right) \frac{\partial}{\partial \hat{x}^{k}}+\frac{1}{2} \sum_{k, l=1}^{M+m} \sum_{j=1}^{M+m} \hat{b}^{k, j}\left(X_{t}\right) b^{l, j}\left(X_{t}\right) \frac{\partial^{2}}{\partial \hat{x}^{k} \partial \hat{x}^{l}}$,

$G^{j}=\sum_{k=1}^{M+m} \hat{b}^{k, j}\left(X_{t}\right) \frac{\partial}{\partial \hat{x}^{k}}$,

where the lower case represents the elements of matrices listed in eqn (A.1). The high dimensional stochastic integrals in eqn (16) may be simplified in a weak sense in terms of three point and two point random numbers. ${ }^{20}$ Operating with $G^{0}$ on $\hat{A}$ up to index $M$ gives $G^{0} \hat{A}=\left[-i L^{I}\left(\Omega_{t}\right) \rho\left(\Omega_{t}\right)\right]\left[-i L_{I}\left(\Omega_{t}\right)\right]$, and no $G^{0}$ operator needs to be listed in eqn (15). Since there is a parametric dependence in the SLEL on a stochastic process, the expansion of the SLEL part [cf. eqn (15)] takes a deterministic form and the necessity of treating the whole problem with Itô calculus may be questioned. However, this approach provides useful insights that enable the construction of an efficient scheme for the whole problem as well as a valid error estimator in the Itô sense.

\section{B. Appendix}

The spin Hamiltonian is in a generally applicable format expressed in spherical tensor notation as follows: ${ }^{48}$

$$
\begin{aligned}
H\left(\Omega_{t}\right) & =\sum_{\mu} F_{\mu}^{(0,0) *} A_{\mu}^{(0,0)}+\sum_{\mu, m} F_{\mu, L}^{(2, m) *}\left(\Omega_{t}\right) A_{\mu, L}^{(2, m)}, \\
F_{\mu, L}^{(2, m)}\left(\Omega_{t}\right) & =\sum_{m m^{\prime}} D_{m m^{\prime}}^{2 *}\left(\Omega_{t}\right) F_{\mu, P}^{\left(2, m^{\prime}\right)},
\end{aligned}
$$

where $F_{\mu, \eta}^{(l, m)}$ and $A_{\mu, \eta}^{(l, m)}$ are proportional to the irreducible spherical tensor operators, $\eta=\{L, P\}$ are the Lab and Principal references frames respectively, $D^{2}$ are the Wigner rotation matrix elements of second $\operatorname{rank},{ }^{49} \Omega_{t}=\{\alpha, \beta, \gamma\}$ are the Euler angles for the $L \rightarrow P$ transformation and $\mu$ is the type of interaction $g_{n}, \mathrm{~g}, A_{i}$ (nuclear-, electron-Zeeman and the hyperfine interaction for nucleus $i=1,2,3)$. We consider only isotropic nuclear Zeeman interaction from, in the case of multinuclear system, the $\mathrm{Cu}(\mathrm{II})$ nucleus with spin Hamiltonian

$$
F_{g_{n}}^{(0,0)}=-\frac{2 \pi \mu_{n}}{h} g_{n}, \quad A_{g_{n}, L}^{(0,0)}=B_{0} I_{z},
$$

where $\mu_{n}, h, g_{n}$ and $I_{z}$ are the nuclear magneton, Planck's constant, isotropic chemical shift, and nuclear spin operator respectively. The components of the irreducible electron Zeeman (magnetic) tensor in principal frame $(P)$ are

$$
\begin{aligned}
& F_{g}^{(0,0)}=-\sqrt{\frac{1}{3}} \frac{2 \pi \beta_{e}}{h}\left(g_{x x}+g_{y y}+g_{z z}\right), \\
& F_{g, P}^{(2,0)}=\sqrt{\frac{2}{3}} \frac{2 \pi \beta_{e}}{h}\left(g_{z z}-\frac{g_{x x}+g_{y y}}{2}\right), \\
& F_{g, P}^{(2, \pm 1)}=0, \\
& F_{g, P}^{(2, \pm 2)}=\frac{1}{2} \frac{2 \pi \beta_{e}}{h}\left(g_{x x}-g_{y y}\right),
\end{aligned}
$$

where $\beta_{e}$ is the Bohr magneton. The Zeeman spin operators are

$$
\begin{aligned}
A_{g, L}^{(0,0)} & =-\sqrt{\frac{1}{3}}\left(B_{0} S_{z}\right), \\
A_{g, L}^{(2,0)} & =\sqrt{\frac{2}{3}}\left(B_{0} S_{z}\right), \\
A_{g, L}^{(2, \pm 1)} & =\mp \frac{1}{2}\left(B_{0} S_{ \pm}\right), \\
A_{g, L}^{(2, \pm 2)} & =0 .
\end{aligned}
$$

For Hyperfine interaction the tensor operators are given by:

$$
\begin{aligned}
F_{A_{i}}^{(0,0)} & =-\sqrt{\frac{1}{3}} \frac{2 \pi \beta_{e} g_{e}}{h}\left(A_{i x x}+A_{i y y}+A_{i z z}\right), \\
F_{A_{i}, P}^{(2,0)} & =\sqrt{\frac{2}{3}} \frac{2 \pi \beta_{e} g_{e}}{h}\left(A_{i z z}-\frac{A_{i y y}+A_{i z z}}{2}\right), \\
F_{A_{i}, P}^{(2, \pm 2)} & =0 \\
F_{A_{i}, P}^{(2, \pm 2)} & =\frac{1}{2} \frac{2 \pi \beta_{e} g_{e}}{h}\left(A_{i y y}-A_{i z z}\right),
\end{aligned}
$$

in Principal frame for nucleus $i$ and $g_{e}$ is free electron $g$ value. The corresponding spin operators are:

$$
\begin{aligned}
A_{A_{i}, L}^{(0,0)} & =-\sqrt{\frac{1}{3}}\left(I_{i z} S_{z}+\frac{1}{2}\left(S_{+} I_{i-}+S_{-} I_{i+}\right),\right. \\
A_{A_{i}, L}^{(2,0)} & =\sqrt{\frac{2}{3}}\left(I_{i z} S_{z}-\frac{1}{4}\left(S_{+} I_{i-}+S_{-} I_{i+}\right)\right. \\
A_{A_{i}, L}^{(2, \pm 1)} & =\mp \frac{1}{2}\left(S_{ \pm} I_{i z}+S_{z} I_{i \pm}\right) \\
A_{A_{i}, L}^{(2, \pm 2)} & =\frac{1}{2}\left(S_{ \pm} I_{i \pm}\right) .
\end{aligned}
$$


In the EPR application we use cylindrical symmetric magnetic tensors (i.e. $\mathrm{g}_{\|}=\mathrm{g}_{x x}=\mathrm{g}_{y y}, \mathrm{~g}_{\perp}=\mathrm{g}_{z z}$ ) with typical Cu(II)-square planar complex values (similar to ref. 43) listed in Table 3. A nonsecular approximation is commonly taken as treating $S_{ \pm}$in hyperfine part eqn (B.17)-(B.19) approximatively. ${ }^{6}$ The operator

$$
A_{A_{i}, L}^{(0,0)}
$$

is put in a secular approximation in the numerical studies.

\section{Acknowledgements}

Grant sponsor: United Kingdom Engineering and Physical Sciences Research Council; contract/grant number: EP/F006802/1. The authors acknowledge the use of the IRIDIS High Performance Computing Facility, and associated support services at the University of Southampton, in the completion of this work.

\section{References}

1 D. Gamliei and H. Levanon, Stochastic Processes in Magnetic Resonance, World Scientific Publishing Co. Pte. Ltd., 1995.

2 R. Kubo, J. Math. Phys., 1963, 4, 174.

3 Y. Tanimura, J. Phys. Soc. Jpn., 2006, 75, 082001.

4 D. Schnider and J. Freed, Adv. Chem. Phys., 1989, 73, 387.

5 N. Usova, P.-O. Westlund and I. Fedchenia, J. Chem. Phys., 1995, 103, 96-103.

6 R. F. Campbell and J. H. Freed, J. Phys. Chem., 1980, 84, 2668-2680.

7 K. Åman, P. Håkansson and P.-O. Westlund, Phys. Chem. Chem. Phys., 2005, 7, 1394-1401.

8 P. Håkansson and P.-O. Westlund, Spectrochim. Acta, Part A, 2005, 61, 299-304.

9 M. Itzkowitz, J. Chem. Phys., 1967, 46, 3048-3056.

10 J. B. Pedersen, J. Chem. Phys., 1972, 57, 2680-2683.

11 H.-J. Steinhoff and W. L. Hubbell, Biophys. J., 1996, 71, 2201-2221.

12 H. Eviatar, E. van Fassen and Y. Levine, Chem. Phys. Lett., 1992, 195, 233-238.

13 B. Robinson, L. Slutsky and F. Auteri, J. Chem. Phys., 1992, 96, 2609.

14 D. Sezer, J. H. Freed and B. Roux, J. Chem. Phys., 2008, 128, 165106.

15 V. S. Oganesyan, Phys. Chem. Chem. Phys., 2011, 13, 4724 4737.

16 K. Aman and P.-O. Westlund, Phys. Chem. Chem. Phys., 2007, 9, 691-700.

17 S. M. Abernathy and R. R. Sharp, J. Chem. Phys., 1997, 106, 9032-9043.

18 R. Kosloff, J. Phys. Chem., 1988, 92, 2087-2100.

19 K. Åman and P.-O. Westlund, Mol. Phys., 2004, 102, 1085-1093.

20 P. E. Kloeden and E. Platen, Numerical Solution of Stochastic Differential Equations, Springer, 1992.

21 P. Håkansson, P.-O. Westlund, E. Lindahl and O. Edholm, Phys. Chem. Chem. Phys., 2001, 3, 5311.
22 N. Usova, L. Persson and P.-O. Westlund, Phys. Chem. Chem. Phys., 2000, 2, 2785-2793.

23 S. Mukamel, Principles of Nonlinear Optical Spectroscopy, Oxford University Press, 1995.

24 L. Persson, U. Cegrell, N. Usova and P.-O. Westlund, J. Math. Chem., 2002, 31, 65-89.

25 P. Håkansson, L. Persson and P.-O. Westlund, J. Chem. Phys., 2002, 117, 8634-8643.

26 P. Håkansson and M. Mella, J. Chem. Phys., 2007, 126, 104106.

27 P. Håkansson, M. Isaksson, P.-O. Westlund and L. B.-A. Johansson, J. Phys. Chem. B, 2004, 108, 17243-17250.

28 P.-O. Westlund, P. Håkansson and L. Persson, Recent Res. Devel. Chem. Physics, (4), Transworld Research Network, 2003, pp. 533-559.

29 D. Talay and L. Tubaro, Stochastic Anal. Appl., 1990, 8, 483-509.

30 E. Platen, Math. Comput. Simul., 1995, 38, 69-76.

31 Y. Saad, Iterative Methods for Sparse Linear Systems, 2nd edn, SIAM, Philadelphia, 2003.

32 S. Balay, K. Buschelman, W. D. Gropp, D. Kaushik, M. G. Knepley, L. C. McInnes, B. F. Smith and H. Zhang, PETSc Web page, 2009, http://www.mcs.anl.gov/petsc.

33 S. Balay, K. Buschelman, V. Eijkhout, W. D. Gropp, D. Kaushik, M. G. Knepley, L. C. McInnes, B. F. Smith and H. Zhang, PETSC Users Manual, Argonne National Laboratory Technical Report ANL-95/11-Revision 3.0.0, 2008.

34 S. Balay, W. D. Gropp, L. C. McInnes and B. F. Smith, Modern Software Tools in Scientific Computing, 1997, pp. 163-202.

35 S. S. Eaton, R. Eaton, Gareth, J. Berliner and Lawrence, in Biomedical EPR-Part A: Free radicals, Cluwer academic/Plenium publishers, metals, medicine and Physology, 2005, ch. 13.

36 mPackages are a set of routines compatible with Mathematica 7.0 (wolfram) programed by Levitt, M. H., available on line at: http://www.mhl.soton.ac.uk/.

37 P.-O. Westlund and H. Wennerström, J. Chem. Phys., 1993, 99, 6583-6589.

38 M. Isaksson, P. Hägglöf, P. Håkansson, T. Ny and L.-B. Johansson, Phys. Chem. Chem. Phys., 2007, 3914-3922.

39 V. B. Cheng, J. Henry, H. Suzukawa and M. Wolfsberg, J. Chem. Phys., 1973, 59, 3992-3999.

40 S. Stoll and A. Schweiger, J. Magn. Reson., 2006, 178, 42-55.

41 S. Stoll and A. Schweiger, Biol. Magn. Reson, 2007, 27, 299-321.

42 G. Della Lunga, R. Pogni and R. Basosi, J. Phys. Chem., 1994, 98, 3937-3942.

43 M. Pasenkiewicz-Gierula, W. Subczynski and W. Antholine, J. Phys. Chem. B, 1997, 101, 5596-5606.

44 G. Della Lunga, M. Pezzato, M. Camilla Baratto, R. Pogni and R. Basosi, J. Magn. Reson., 2003, 164, 71-77.

45 L. D. Favro, Phys. Rev., 1960, 119, 53-62.

46 M. B. Giles, Operations Research, 2008, 56, 607-617.

47 C. S. Burns, E. Aronoff-Spencer, G. Legname, S. B. Prusiner, W. E. Antholine, G. J. Gerfen, J. Peisach and G. L. Millhauser, Biochemistry, 2003, 42, 6794-6803.

48 P. L. Nordio, in General Magnetic Resonance Theory, Academic Press, Inc, 1976, ch. 2, pp. 5-52.

49 D. M. Brink and G. R. Satchler, Angular Momentum, Oxford University Press, 1993. 